\title{
Risk of Regrettable Substitution under EU REACH: Level Playing Field in the EU Regulatory Context
}

\author{
Dieter Drohmann and Francisco Hernández*
}

The substitution process is an important tool defined in the REACH Regulation to remove the risks derived from the use of hazardous chemicals. However, when the alternative substance is suitable in technical and economic terms, but it is just as harmful or potentially worse as the replaced one, the process results in regrettable substitution. The absence of information regarding the hazard properties of the substitute substance, inconsistencies on implementation of the European chemicals Regulations, and the lack of interest of some part of the industry to manage stringent classifications, are the main reasons why this situation is generated. Change in the paradigm 'No data, no market' to 'No data, no problem', and replacement with the 'Least regulated alternative' instead of the 'Least hazardous alternative' are the results of the regrettable substitution scenarios. In order to avoid this practice, EU Authorities and the chemical industry should work together to achieve solutions, like clarifying the objective of substitution, improving the regulatory processes, or involving industry and stakeholders into rigorous evaluations of potential alternatives to substances of concern. This will guarantee a level playing field for companies on the European market of chemicals in front of regrettable substitution.

\section{Introduction}

Article 1 of the REACH Regulation (1907/2006/EC) ${ }^{1}$ outlines the three main objectives of this important piece of European Legislation on the management of chemicals in the EU: to ensure a high level of protection of human health and the environment, to guarantee the free circulation of chemicals on the internal European market, and to enhance the competitiveness and the innovation of the European chemicals industry. To achieve these goals, the REACH Regulation establishes different mechanisms focused on the increase (quantity and quality) and the dissemination of the information and knowledge on chemi-

DOI: $10.21552 / \mathrm{icrl} / 2020 / 1 / 6$

* Dieter Drohmann is CEO of the Chemservice Group; E-Mail: <d .drohmann@chemservice-group.com>; Francisco Hernández is Regulatory Affairs Advisor at Chemservice Iberia; E-Mail: $<\mathrm{f}$ .hernandez@chemservice-group.com>

1 Available at: <https://eur-lex.europa.eu/legal-content/EN/TXT/?qid $=1588673855274 \&$ uri $=$ CELEX:32006R1907 $>$ accessed 15 May 2020. cals (Registration, Evaluation and Communication in the supply chain), and to promote substitution of chemicals classified as hazardous on the basis of their health, environmental or physical effects, particularly for those that are considered as Substances of Very High Concern (SVHC), via the Authorisation and Restriction processes.

Although these two mechanisms have been developed to achieve the same objectives, both have different scopes. Restriction is focused on stablishing conditions for safe use, limiting, or banning the manufacture, the marketing and the use of chemicals of concern that are not adequately controlled and which pose an unacceptable risk, requiring regulatory action on a Community wide basis. Authorisation is based on the substitution of SVHCs by suitable alternatives when those alternatives are technically and economically available. Alternatives could be single chemical substances, combinations of substances, or the use of different technologies or processes.

It is important to point out that substitution is a process that is not strictly defined and structured in 
the legal text of the REACH Regulation. It is covered only as an activity inside the major mechanism of Authorisation. Also, the legal text (Article 55) refers to the alternative substance as being 'suitable' not only in terms of risk, but also in terms of technical and economic feasibility. The absence of clearly defined rules to manage the substitution process, and the fact that the Regulation is bringing to the same level the risk, the technical and the economic aspects of the alternative, can lead to the replacement of a chemical by a similar (or possibly worse) chemical in terms of hazards or risks. In this case, a situation of regrettable substitution can be generated.

Regrettable substitution leads to a non-level playing field in the European chemicals market. When a substance is replaced by another chemical which ultimately leads to equal or higher levels of hazard or risk, the Regulation is introducing a discriminatory factor on the manufacturers and importers of the replaced substance, because this leads to a loss of market share in front of a substitute substance that does not show any advantage in terms of protection to human health and the environment.

Moreover, it is necessary to highlight that the starting point of any discussion around hazard, risk or substitution should not be related to REACH, but to the CLP Regulation. Substances need to be classified and labelled following the principles of CLP. Typically, the basis for implementation of CLP by industry comes from data generated in the REACH registration process. However, there are a number of practical gaps in the implementation of CLP that are already leading to situations of regrettable substitution in Europe. Some examples of this situation, which will be discussed further, are:

- Limited enforcement from CLP obligations, which basically allows free-riders to place their products on the market without having performed a proper evaluation of their substances. This need for improved enforcement practices should include not only substances with harmonised classifications listed in Annex VI of CLP, but also agreed conclusions by industry that come from extensive data generation and internal discussions within SIEF and/or REACH consortia.

- Exemptions under REACH, for example in the case of naturally occurring substances; if these substances are not registered, and since the main source of information to establish classification and labelling is typically data coming from the reg- istration dossier, a door is open for the suppliers of those materials to market them without a thorough assessment of their properties.

- Limited use of the C\&L Inventory, which contains information provided by stakeholders on how chemicals should be classified, and which may provide justifications to further investigate on the adequate classification and labelling of certain chemicals.

\section{The Authorisation Process - from Classification to REACH Annex XIV, via SVHC listing}

As previously stated, substitution is only discussed in the REACH legal text in the context of authorisation. However, this does not reflect the reality of how the markets of certain chemicals behave. When a given chemical is classified, either via harmonised classification, or because of a decision by industry (eg via new findings related to REACH registration), suppliers of (potential) alternative substances may use this situation to promote their products as safer replacements. This is particularly significant in the case of properties that may trigger identification of a chemical as a SVHC, which frequently leads to 'stigmatisation' of the impacted chemicals. If those alternative substances are not subject to a thorough process to clearly establish their correct classification and labelling, regrettable substitution may occur even years before a substance is considered for authorisation or even for inclusion in the candidate list.

This situation is related to the duration of the $\mathrm{Au}$ thorization process, and the different steps ${ }^{2}$ that can be identified. Those steps have a certain number of fixed actions that require certain timelines that need to be observed. In general terms, three different phases can be identified in the process, that can take up to five years to completion. However, since the final decision to include a substance in REACH Annex $\mathrm{XIV}$ is on the European Commission (COM), this could be even longer, if for whatever reason, COM decides to not include a substance in the authorization list.

2 Available at: <https://echa.europa.eu/es/authorisation-process > accessed 15 May 2020 
The first phase is the identification of the substance as an SVHC and its inclusion in the Candidate List $^{3}$, this starts with the submission of an Annex XV dossier (SVHC dossier) by a Member State Competent Authority (MSCA) or by ECHA (after the request of $(\mathrm{OM})$. It has to be highlighted that there is no clear established procedure to indicate when a substance may be taken up for inclusion in the Candidate List. It is a decision of the Authorities, that depends on the knowledge about the substance, or on the existing social pressure on the substance.

Moreover, in application of Article 57 of the REACH Regulation, only substances with specific intrinsic properties can be considered for inclusion in the candidate list. Those properties are:

- Carcinogenic, Mutagenic or Toxic to Reproduction (CMR) category $1 \mathrm{~A}$ or $1 \mathrm{~B}$,

- Persistent, Bio-accumulative and Toxic (PBT) or very Persistent and very Bio-accumulative (vPvB),

- Those identified, on a case-by-case basis, from scientific evidence as causing probable serious effects to human health or the environment of an equivalent level of concern as those above. This may include, among others, Endocrine Disruptors (ED), skin or respiratory sensitizers, or Specific Target Organ Toxicants following Repeated Exposure (STOT-RE).

The SVHC identification process is divided in four different actions: the registration of the SVHC intention until outcome (ROI), the preparation of the SVHC dossier, a consultation period, and the addition of the substance to the Candidate List. The SVHC dossier must outline the scientific evidence for identifying the substance as a SVHC, it must be prepared according to the requirements given in Annex XV of $\mathrm{REACH}$, and it should include the data and justification for identifying the substance as SVHC, jointly with further information relevant for the follow-up process (the uses, volumes and possible alternatives to the substance $)^{4}$.

Once ECHA has assessed the proposal, and if the document passes the accordance check, the SVHC dossier will be published on the ECHA website, and

3 Available at: https://echa.europa.eu/candidate-list-table accessed 15 May 2020.

4 Available at: <https://echa.europa.eu/documents/10162/13638/ annex_xv_svhc_report_template_en.doc/> accessed 15 May 2020. a public consultation is launched, in which Member States and interested parties can participate. All these stakeholders are invited to submit comments or provide further information mainly related to the identity of the substance and its hazardous properties. Comments about uses, volumes per use, exposure, alternatives and risks of the substance, will be taken into account in later steps of the authorization process. The substance will be added to the Candidate List (which will be updated and published by ECHA) if:

- No comments are received challenging the identification as SVHC

- Comments are received, and the Member States Committee (MSC) decides by unanimous agreement that the substance should be identified as an SVHC after assessment of the information received,

- COM decides that the substance has to be identified as an SVHC, after receiving the matter from the MSC after a non-unanimous agreement

This phase could last at least one year and, once a substance is included in the Candidate List, it brings immediate obligations for its suppliers, such as the need to provide their customers with a Safety Data Sheet (SDS), for pure substances and for mixtures which contain more than $0.1 \%$ by weight of the SVHC. This means that customers are directly informed about the change in the legal situation of the substance, which is frequently the first incentive to look for alternatives.

The second phase of the authorization process is the recommendation for inclusion in the Authorization List (REACH Annex XIV), also known as prioritization. This is another relatively long action which may take up to one year and a half. This phase is divided in five different actions: prioritisation, preparation of the draft recommendation, consultation period, submission of the MSC opinion, and recommendation and inclusion in the Authorization List.

The first action is the prioritisation, by which ECHA develops a list of substances from the Candidate List that should be moved to REACH Annex XIV. In this step, the information in the registration dossier is analysed, together with other sources of information such as the input provided by stakeholders (eg industry) during the public consultation launched on the SVHC identification phase. The prioritization is justified by ECHA in a draft recommen- 
dation document on the basis of intrinsic properties of the substance, volumes manufactured or imported, and types of uses. The different substances evaluated receive a specific scoring depending on the severity of those criteria. In the recommendation provided by ECHA, the following information is also included: the latest application date ${ }^{5}$ (LAD), the sunset date $^{6}$, the recommended review periods for certain uses, and the uses exempted from the authorisation requirement.

The scoring criteria are based on inherent properties (eg PBT or vPvB gets 13 points), volume within the scope of the authorisation $(\mathrm{eg}>10.000 \mathrm{t} / \mathrm{a}=15)$ and wide dispersive use (eg consumer use $=15)$. Further details are available at the ECHA Guidance Documentation. $^{7}$

The publication of the list of substances that will be prioritised is followed by another public consultation. It should be noted that comments taken into consideration by ECHA in this consultation are related almost exclusively to the Authorisation List entries, the complexity of the supply chain, the exempted uses and the information of volumes and uses. Comments related to alternative substances are not considered by ECHA in this step of the process. This information is taken into account in the phases of application and Authorisation decision, once the substance is included in the Authorisation List, as it is clearly stated in the document 'ECHA's general responses on issues commonly raised in consultations on draft recommendations ${ }^{\prime 8}$. All the comments received during the public consultation are considered in an opinion document on the draft recommendation, prepared by the MSC and sent to ECHA, with the purpose to help ECHA to finalise the recommendation. Finally, ECHA submits the final recommendation to COM, which takes together with the REACH Committee $^{9}$ the final decision on the substances to be included in the Authorisation List. The decision is the last action in this phase, and it is published in the Official Journal of the EU and the Authorisation List is updated on ECHA's website ${ }^{10}$. The REACH Regulation is updated as well, with the modification of Annex XIV.

From this point, companies must apply for an authorisation to continue the use and placing the substance on the market before the LAD. This process, that can take at least two years, will include the evaluation of alternative substances for the substitution of the SVHCs, as indicated in the REACH legal text.

\section{What is Regrettable Substitution}

As previously discussed, regrettable substitution occurs when a chemical is replaced by an alternative substance (or substances) that is (are) just as harmful as the replaced one, or potentially worse. There are different reasons why regrettable substitution takes place, but the most important one is probably the lack of information about the alternative substance. Typically, substances that are included in the pipeline for regulatory scrutiny (starting with classification, SVHC listing and so on) are well-known substances that have been extensively studied, either via REACH or through previous assessments. In most cases, alternatives are substances that have not been thoroughly evaluated, with limited information on toxicity and ecotoxicological data on the substance itself. Frequently the lack of data on a chemical is perceived as being 'less hazardous' by industry, when this may actually not be the case (if there is no data, then there is no risk). As soon as the substitute is under scrutiny and new data is being generated, very often the substitute itself enters the substitution route via SVHC and Annex XIV listing and regretable substitution occurs.

In many cases it is predictable that alternatives have the same intrinsic properties and toxic profiles, because the functionality and technical properties of the chemical in a certain application just requires it. Many chemicals are designed to be Persistent $(\mathrm{P})$ and Bioaccumulative (B) in order to be stable and not water soluble. These are properties biological systems dislike and therefore often ' $\mathrm{T}$ ' (Toxic) is associated too.

In parallel, there are different reasons why, even 10 years after the first REACH registration deadline,

5 A date or dates, at least 18 months before the sunset date(s), by which applications for authorisation must be submitted if the applicant wishes to continue to use the substance or place it on the market for certain uses after the sunset date(s) until a decision on the application for authorisation is taken.

6 Date from which the placing on the market and use of a substance is prohibited, unless an authorisation is granted or the use is exempt from authorisation.

7 Available at: <http://echa.europa.eu/documents/10162/13640/gen _approach_svhc_prior_in_recommendations_en.pdf>

8 Available at: <https://echa.europa.eu/documents/10162/13640/ recom_general_responses_doc_en.pdf/44e192e5-ac72-4458-b4f5 -c016754a1d4c> accessed 15 May 2020.

9 Available at: <https://www.eumonitor.eu/9353000/1/ j9vvik7m1c3gyxp/vhspovzauvof> accessed 15 May 2020.

10 Available at: <https://www.echa.europa.eu/authorisation-list> accessed 15 May 2020. 
there are still substances in the EU market that have not been adequately investigated. As previously discussed, inconsistencies between implementation of REACH and CLP may be among the main reasons for this situation. In addition, some procedures appear to be rather slow, and not always focusing on alternatives of substances of concern, eg dossier / substance evaluation or resolution of testing proposals that could clarify data gaps. Furthermore, divergent criteria for evaluation of the available information by industry may be behind this situation. For example, read-across principles are not always being used consistently through different sectors. For some chemicals that have not been covered by relevant REACH consortia, which could be chemically similar and technically equivalent to a hazardous substance, read across to the classification of the original chemical may be relevant. However, this may be ignored by the suppliers of the alternative, just in order to avoid a stringent classification. Because those situations typically escape the radar of enforcement authorities, the paradigm 'No data, no market', one of the most important pillars of the REACH Regulation, is changed to 'No data, no problem' for the suppliers of these potentially hazardous substances.

There are limited legal options for an industry or group of industries to convince suppliers of alternative chemicals that their hazard assessment may not be sufficiently robust. In addition, it has to be underlined that for certain downstream users, the possibility of using a non-classified chemical is very tempting, and usually preferred, as this is seen as a commercial advantage. This frequently leads many users to not asking the right questions to their suppliers, or not digging deeply enough on the reasons why a chemical has not been assigned a certain classification. A reality that is being observed in certain sectors, is that downstream users of chemicals do not substitute a chemical of concern with the least hazardous alternative', but with the 'least regulated alternative', this being another clear example of regrettable substitution.

\section{Case Studies}

The following examples describe specific situations with chemicals or groups of chemicals, for which regrettable substitution is (or may be) already occurring in the EU.

\section{Bisphenol-A Case}

The hormone-disrupting chemical bisphenol-A (BPA), has been banned for use in baby bottles and other plastic products. However, this may not have completely removed risks for consumers, because BPA may have been replaced by bisphenol-S (BPS), a similar chemical which may be even more harmful to children's health.

BPA (4,4'-isopropylidenediphenol - EC 201-245-8, CAS $80-05^{-7}$ ) is regulated in the EU market in compliance with REACH and CLP Regulations. Although suspicion about its behaviour as an ED dates back many years, this substance was not classified as SVHC until 2017. An ED substance is a chemical that interferes with the hormone system of humans causing, even in very low doses, permanent changes in hormonal or reproductive abilities, and affecting the normal development of children. BPA has a specific entry in Annex VI of CLP (harmonised classification), since the approval of the $9^{\text {th }}$ adaptation to technical and scientific progress (ATP) of the CLP Regulation, as Reprotoxic Category $1 \mathrm{~B}$ (Repr. 1B). The use of BPA has been banned in baby bottles since 2011 and will be prohibited in thermal paper starting in 2020.

BPA is part of a large family of synthetic organic compounds (named bisphenols) formed by approximately 40 similar chemicals. They are mainly used to manufacture plastics and epoxy resins and, for this reason, they are found in many consumer products that we use every day; food and drink packaging (such as water and infant bottles, cans), DVDs and CDs, toys and automotive parts. They are also present in thermal paper, medical equipment, and some dental sealants. It is suspected that, due to their similar structure and chemical behaviour, the entire bisphenol family may share common ED properties.

BPS (4,4'-sulphonyldiphenol - EC 201-250-5, CAS 80-09-1) is part of the bisphenols family and, although it is subject of the same legislations than BPA, there is less information available on it. BPS is registered according to the REACH Regulation, but its ED assessment is still under development and the substance is not included in the Candidate List of SVHCs. Regarding the CLP Regulation, there is a proposal for harmonised classification as Repr. $1 \mathrm{~B}$, but it is still under development. This means that there is no clear and final conclusion that the substance will not have a similar toxic profile to BPA. 
However, BPS is currently used as an alternative substance for the use of BPA, and it is a component of many plastic products marketed as BPA-free. Substitution is occurring because BPS has similar technical properties to BPA. Although there is not full scientific certainty and evaluations are on-going, it is not unreasonable to expect that BPS may exhibit similar ED effects as BPA. In summary, manufacturers of the above-mentioned products may be taking advantage of the lack of information and the lower regulatory pressure on BPS compared to that on BPA, which may result in potentially regrettable substitution of BPA. This is a clear example of substitution with the least regulated alternative.

\section{Antimony Trisulfide Case}

Antimony trisulfide (ATS - EC 215-713-4, CAS 1345-04-6) is a substance that can be manufactured from antimony metal or other antimony compounds. It is also a naturally occurring mineral, and it can be placed as such on the market, with no further chemical processing. It is supplied as a black powder, and it is mainly used in brake pads, friction materials and as primer in ammunitions.

ATS was registered under the REACH Regulation in 2013, and the dossier has been regularly updated (latest update issued on 2019). Two co-registrants in the EU have participated in the registration, these are companies that manufacture the synthetic substance. As part of their REACH obligations, they have evaluated the toxicological data available for the substance, and they have also generated new data where deemed relevant. The co-registrants have concluded that ATS requires a self-classification under CLP as Carc. 2 (inhalation) and STOT RE 2 (lung). There is currently no specific entry for ATS in Annex VI of CLP; a generic 'antimony compounds' entry is included in Annex VI; however, this explicitly excludes ATS.

There are other suppliers of ATS in Europe which supply the natural product (ore), mainly imported from China. Because natural ATS is not subject to registration (due to the exemption from registration of naturally occurring minerals in Annex V of REACH), those suppliers do not need to participate in the discussions that the registrants of the synthetic ATS have conducted. When they have been approached by the relevant industrial association supervising regulatory affairs for ATS in EU (including the work done for registration), suppliers of natural ATS typically question de work performed under REACH, and also that such work should be relevant for the ATS they introduce in the EU market, however, they do not provide any substantial alternative toxicological data, or a justification for the lack of classification and labelling

It is also worth noting that 163 notifications of ATS can be found in the ECHA Classification and Labelling (C\&L) Inventory. In this case, most notifications report the classification information from the above-mentioned generic entry for antimony compounds in CLP Annex VI, however as previously stated this is not correct as this entry does not apply to ATS $^{11}$.

This situation generates a distortion in the ATS market, but it may also lead to a situation in which downstream users may be exposed to unacceptable levels of ATS, because they may not be receiving adequate information related to its properties and adequate handling instructions ${ }^{12}$. Indeed, suppliers that do not assign a classification to the ATS they distribute are not delivering an SDS with adequate communication of the properties that ATS may exhibit. Relying on the more frequent C\&L notifications in ECHA's inventory may also lead to confusing conclusions.

Unfortunately, it is common practice for DUs to prefer sourcing a material from a supplier that does not provide a classification to their products rather than from suppliers that do classify the same substance. Frequently, DUs are not aware of these divergent positions, or if they are, they do not want to ask questions that would be relevant to get a clarification of the situation. Most of the times DUs are happy with simply receiving a statement from the supplier of the non-classified material, without going into an in-depth analysis of whether the reply received is scientifically solid. The result is that responsible suppliers are unable to influence customers and lose market, while they need to face additional regulatory pressure coming from their responsible behaviour (eg evaluation procedure of ATS under CoRAP), and DUs may expose workers to unsafe levels of ATS.

11 These notifications may be related to other antimony impurities present in ATS; however, the C\&L inventory does not report if this is the case and therefore this cannot be verified.

12 It is assumed that self-classification agreed by REACH co-registrants is likely the most reliable source of information related to potential hazards of ATS, as this is coming from expert judgment after evaluation of the toxicological data under REACH. 
The ATS case is a clear example of regrettable substitution based on the paradigm of 'No data - no problem', derived from the exemption of Registration of the natural minerals under the REACH Regulation.

\section{Borates Case}

Borates are substances that contain boron in their chemical composition. In these substances, boron is frequently bonded to oxygen atoms forming a borate anion, which can be united to an alkali/alkali-earth cation, being boric acid (EC 233-139-2, CAS 10043-35-3) and boric oxide (EC 215-125-8, CAS 1303-86-2) the simplest forms of borates. Borates can be obtained as naturally occurring minerals from the earth crust. Those minerals typically require a refining process to obtain products that meet the boron content demand by industry.

These chemicals have a wide range of uses: adhesives and sealants, fertilizers, batteries and capacitors, ceramics, enamels, and glazes, cleaners and detergents, fire retardancy materials, glass and textile fiberglass, industrial fluids and lubricants, insulation and polymers, nuclear energy, oil and gas production, pharmaceuticals, wood protection and biocides.

The hazard profile of borates has been extensively discussed at EU level over the past twenty years, related to potential adverse effects on development and fertility in humans. An extensive dataset of scientific studies exists that shows reprotoxic effects in animals from different borates, mainly boric acid, when exposed to very high doses. In contrast, other human studies are also available, conducted on borate mining workers (presumably in the realistic worst-case exposure scenarios for humans), which have not shown any reprotoxic effects.

The main borate substances boric acid, boric oxide, and disodium tetraborates (EC 215-540-4, CAS 1330-43-4 / 12179-04-3 / 1303-96-4) were included in the $1^{\text {st }}$ ATP of the CLP Regulation with harmonised classification as Repr. 1B, and disodium octaborate (EC 234-541-0, CAS 12008-41-2 / 12280-03-4) was included in the $9^{\text {th }}$ ATP with the same classification Moreover, at present time there are some open regulatory initiatives on other registered borate substances, in addition to a revision of the Specific Concentration Limits that were originally included in CLP for the main borates. It is important to note that this position on classification and labelling of borates as reprotoxic substances, together with the seemingly strong regulatory pressure that EU authorities are placing on refined borates, does not impact the borate minerals (basically colemanite - LN 817-854-2 , CAS 1318-33-8 / 12291-65-5, and ulexite - LN 603-535-3, CAS 1319-33-1), that are being placed on the EU market as non-hazardous materials, therefore without any kind of hazard classification.

This lack of classification of borate minerals is directly related to the lack of information about the hazard profile of the substances themselves. As naturally occurring minerals, they are exempted from the obligation to register under the REACH Regulation (according to point 7 of Annex V). For this reason, suppliers of borate minerals have not been involved in any REACH activities related to evaluating intrinsic properties from these chemicals. However, these substances are not exempted from the obligation to classify and label them under the CLP Regulation.

At present time, there is no specific reproductive toxicity study performed on borate minerals. However, it is to be noted that, in order to adequately establish the classification and labelling of a chemical substance that is under the scope of the CLP Regulation, available data from chemically similar substances should be used, in the absence of specific data for the substance in question, in order to establish classification and labelling conclusions. In this regard, it should be considered that borate minerals are basically borate substances with sodium or sodium/calcium counterions. Indeed, most of the classified borates are obtained through a refining process from the borate minerals. For this reason, it should not be ruled out that borate minerals may have similar hazardous properties if compared to the refined or synthetic borates.

Another relevant source of information when evaluating whether a substance needs to be classified is the information available in the ECHA C\&L Inventory. In this database, borate minerals are reported as reprotoxic materials (91 entries for colemanite and 22 entries for ulexite). Moreover, ulexite is included in the REACH Annex III Inventory. This is a list of substances compiled by ECHA which are predicted as likely to meet criteria for CMR category $1 \mathrm{~A}$ or $1 \mathrm{~B}$ properties. The information source for the inclusion of ulexite in this list is the Repr. $1 \mathrm{~B}$ conclusion established by IMAP (Inventory Multi-tiered Assessment and Prioritisation), under the National Industrial Chemicals Notification and Assessment Scheme 
(NICNAS) from the Department of Health of the Australian Government. Indeed, borate minerals receive a mandatory classification as Repr. 1B in Australia. It appears that in this particular case, Europe is falling behind other regions in terms of better regulation of chemicals.

Taking all the previous information into consideration it would appear that the classification of borate minerals as reprotoxic substances may be justified or, at least, that a thorough hazard assessment should be undertaken by suppliers or Authorities. This situation results in a non-level playing field within the borates market, since borate minerals can be used as substitutes of the classified borates in certain markets because customers tend to prefer unclassified material. In fact, in certain sectors of use, substitution started to occur as early as the date when boric acid and disodium tetraborates were identified as SVHCs and included in the candidate list (2010 and 2011 respectively). It is to be noted that, in spite of being prioritised for authorisation in 2015 , those borates have not yet been moved to the authorisation list. Specific indications have been given by COM in the past, that placing those borates in REACH Annex XIV may create a situation that would be difficult to manage, due to the large number of uses of borates, and therefore a significantly high amount of applications for authorisation would have to be expected. This is a very good example of potential regrettable substitution, which is occurring before authorisation is imposed on a substance, or a group of substances. Again, the registration exemption for minerals, together with the lack of a detailed evaluation of potential alternatives in the early stages of the process, result in a switch of paradigm from 'No data, no market' to 'No data, no problem' for the suppliers of borate minerals.

\section{Terphenyl, Hydrogenated Case}

Terphenyl, hydrogenated (TPH - EC 262-967-7, CAS 61788-32-7) is a substance that is mainly used as a high temperature, non-pressurised heat transfer fluid (HTF) in closed manufacturing systems. It is also used as a plasticizer in some few specific applications.

TPH was registered under the REACH Regulation in 2010, and the dossier has been updated several times (2014, 2018 and 2020). The Finnish MSCA (Tukes) began assessing TPH in 2008. In 2017, via
RMOA, Tukes concluded that the substance should be included in the REACH Candidate List based on $\mathrm{vPvB}$ (very persistent, very bio accumulative) properties. For this reason, TPH was listed as an SVHC in June 2018. However, this substance has not been included in the authorisation List (Annex XIV of REACH Regulation), but it may be considered for the upcoming prioritisation round on 2020 .

There are two substances that could be used as alternatives to TPH as HTFs: Dibenzylbenzene, armethyl derivative (EC 258-649-2, CAS 53585-53-8) and 6-(1-phenylethyl)-1,2,3,4-tetrahydronaphthalene (EC 400-370-7, CAS 6196-98-1). These substances are currently not classified as $\mathrm{vPvB}$ or PBT and they are not listed as SVHCs.

However, in order to meet their functionality requirements for providing product performance over many years, all HTF fluids must possess certain properties that make them likely to meet REACH Annex XIII criteria for persistence and bioaccumulation. This is a feature that is well known to the downstream users of this kind of products. Due to these similar properties, Tukes has finalised an RMOA for the two alternatives to $\mathrm{TPH}^{13}$. This RMOA has been based on the technical functional grouping approach and the potential $\mathrm{vPvB} / \mathrm{PBT}$ properties of these two substances. It does provide indication, if the two alternatives meet as well $\mathrm{vPvB} / \mathrm{PBT}$ properties, they should be regulated at the same level as TPH, which would eventually avoid the risk of regrettable substitution in HTF applications.

Indeed, if the regulatory activities on TPH are not stopped at EU level, and this substance is included in the authorisation list before the assessment of the two alternatives is completed, this could lead to regrettable substitution with its two direct alternatives, damaging competitiveness of the EU industry, with no significant environmental benefit. TPH has currently been prioritized by ECHA to be included in the $10^{\text {th }}$ Recommendation for inclusion into REACH Annex XIV ${ }^{14}$. Currently, a public consultation is ongoing in this regard. This would be an example of forced replacement of a well-know and well-regulated chemical by less-know, less-regulated alternatives.

13 Available at: <https://echa.europa.eu/de/rmoa/-/dislist/details/ 0b0236e1834a0ed6> accessed 15 May 2020

14 Available at: <https://echa.europa.eu/de/recommendation-for -inclusion-in-the-authorisation-list/-/substance-rev/25204/term> accessed 15 May 2020. 


\section{Possible Actions to Prevent Regrettable Substitution}

EU Authorities and the chemical industry have the obligation to take actions to promote the use of safer chemicals in the products that are being placed on the EU market. The substitution process is one of the best strategies to achieve this objective. However, when this process is not properly managed it can lead to situations of regrettable substitution, as it has been explained in the different case studies previously discussed. In order to avoid this, an active approach from both parties is essential: EU Authorities should help to promote a level playing field in the EU chemicals market, by redefining the rules and promoting the right substitution processes; on the other hand, the chemical sector should take a compromise to adequately manage chemicals, involving the whole supply chain.

There are currently some initiatives under development that point towards the right direction. For example, it is worth mentioning the efforts made by ECHA to clarify the objective of the substitution process, and to avoid regrettable substitution, via the publication of the document 'Strategy to promote substitution to safer chemicals through innovation $^{15}$. This document aims at strengthening the relation between the substitution process and the different objectives of the REACH Regulation, since it promotes an improvement to human health and the environment, and it is aligned with the objective to enhance the innovation and the competitiveness of chemical companies, having a positive impact on the implementation of a circular economy ${ }^{16}$ through its emphasis on the need for further research in the chemicals sector.

Another initiative that is worth highlighting comes from the Swedish Chemicals Agency (Kemi), which has published its own document related to 'Grouping of chemical substances in the REACH and CLP regulations ${ }^{\prime 17}$. This approach also highlights the

15 Available at: <https://echa.europa.eu/documents/10162/13630/ 250118_substitution_strategy_en.pdf> accessed 15 May 2020.

16 Available at: <https://echa.europa.eu/substitution-to-safer -chemicals> accessed 15 May 2020.

17 Available at: <https://www.kemi.se/global/pm/2018/pm-2-18 -grouping-of-chemical-substances-in-the-reach-and-clp -regulations1.pdf > accessed 15 May 2020.

18 Available at: <https://echa.europa.eu/documents/10162/966058/ tips_users_chemicals_workplace_en.pdf/> accessed 15 May 2020. capacity of grouping of chemicals (and therefore, covering all the members of a group jointly in regulatory processes) as an efficient way to minimise possibilities of generating situations of regrettable substitution for hazardous chemicals.

Still, additional work would appear to be necessary. This could include, as a starting point, increased emphasis from the regulators' side to use tools and guidance documents that are already available. In this regard, the ECHA short guide 'Tips for users of chemicals in the workplace ${ }^{18}$ might be a good first approach. In this document, ECHA reminds users of chemicals that they have their own responsibilities regarding the hazard information that they receive from suppliers. Indeed, a user of a chemical should not simply give for granted the accuracy of the classification and labelling stated by a supplier, particularly in cases where other suppliers may be using divergent information for the same substance or for structurally similar ones. In these cases, downstream users should ask for clarifications to the suppliers, and if the explanations received are not satisfactory, they should take additional steps, ie requesting support from the ECHA helpdesk.

Another source of information that appears to be underused is the ECHA C\&L Inventory. While it is true that many times it is uncertain whether the information contained in that database is fully reliable (there is no option to verify who has notified a specific classification and labelling, or why), it could help to identify divergent positions for substances of concern, or for potential substitutes for those substances. As described in the previous examples, in some cases using the C\&L inventory as a starting point may help to justify further actions on potential alternatives to hazardous chemicals that may result in regrettable substitution. Related to this, it would also seem advisable for regulators to promote and even enforce the use of self-classifications established by industry, particularly in cases when those come from agreements taken jointly by different actors after evaluation of reliable data (eg in the context of REACH registration). Not having such mechanisms available encourages free-riders to skip their regulatory obligations. These situations are very discouraging for companies that make the effort to comply with their legal obligations, as they often see some competitors taking advantage of this lack of enforcement, by gaining market share promoting their 'nonclassified' products in the market. 
Indeed, it appears that there is still room for improvement on how (or better, when) alternatives are to be considered in the regulatory process for a hazardous substance. It would appear highly advisable that, when a substance is considered for regulatory scrutiny (ie after a Registry of Intentions for establishing harmonized classification, or for inclusion in the candidate list), a thorough investigation of the potential alternatives of the substance should be performed at that stage, leading to an analysis of the hazards and risks related to those alternatives. If the target substance is not evaluated jointly with the alternatives, a risk for regrettable substitution is generated at that point in time, which may lead to a non-level playing field at the EU market. This situation may result in potential increased risks to workers, consumers, or the environment, due to unnoticed exposure to potentially harmful chemicals, and an unfair treatment for the suppliers of the target substance.

Other options could be, for example, to involve different stakeholders in the discussions, eg EU workers unions that could provide a point of view from the direct users of chemicals, in order to engage with them in the debate of handling of chemicals that may be used as replacements of hazardous substances under a given regulatory process.

Regarding the chemicals industry, it also appears to be critical to involve them into rigorous processes and evaluations of their chemicals and their alternatives. There are initiatives on-going in this area, for example the program launched by Eurometaux jointly with ECHA, the MISA (Metals and Inorganics Sectorial Approach) program ${ }^{19}$. This initiative intends to address outstanding technical and scientific issues specifically for the metals and inorganics sectors, with the objective to further improve the registration dossiers in these sectors. It is expected that the work of the involved actors (eg key consortia of metals in EU) will lead to an improvement of data available, read-across principles and grouping possibilities.

Generally speaking, the EU chemicals industry needs to rethink and adapt in front of potential cases of regrettable substitution. In order to comply with the obligation to protect the human health and the environment, suppliers of chemicals that are being marketed without classification, where this position may be questionable, should take a responsible approach to perform a thorough hazard assessment on their products or, when this has actually been conducted, make improved communication efforts with their customers on their conclusions, particularly when limited sources of information exist (eg substances that are exempted from the obligation to register under REACH). It is to be noted that, for chemicals that are not classified by a supplier, there is no obligation to provide a Safety Data Sheet to downstream users, thus resulting in reduced communication obligations. On the other end, downstream users should also place efforts to clarify situations regarding classification of chemicals that may be deemed as doubtful; whereas it is unfortunately the case that some users tend to prefer non-classified materials for marketing and business reasons, those actors should evaluate the unnoticed risks to which they may be exposing their workers when handling chemicals that could be used to replace others with more regulatory pressure. In fact, those companies may be exposing themselves at even higher business risk; for example, if the EU authorities should decide to take action against the alternative in order to avoid regrettable substitution, and they eventually conclude that the alternative should be classified with the same or even more stringent classification than the original substance, the user would then be forced to use that classification. In that event, workers may ask if the company knew about the hazards and risks derived from the use of the alternative substance, if they had placed all efforts to clarify the situation, and what actions the company had taken to protect them. If nonresponsible procedures are identified, this may actually open the ground for lawsuits that the company may need to face. In this regard, highlighting the content of the ECHA guide for use of chemicals at the workplace might appear to be relevant, as previously discussed.

\section{Conclusions}

The substitution of hazardous chemicals is one of the best strategies to reduce the risks derived from the use of chemicals on human health and the environment. Moreover, it is an essential part of key strategies on sustainable development, innovation, and competitiveness of the EU industry. However, when substitution is not adequately managed, there is a se-

19 Available at: <https://www.reach-metals.eu/metals-and-inorganics -sectoral-approach-misa> accessed 15 May 2020. 
rious risk to turn substitution into a source of negative impacts, which no longer meet the objectives for which it was designed.

Regrettable substitution occurs when industry responds to regulatory actions on a hazardous chemical by phasing out its use, and replacing it with an alternative that offers technical equivalence, but introduces similar or even worse properties from the point of view of impacts on human health or the environment. This typically occurs because the alternative has not been studied as thoroughly as the substance that it substitutes, or because it has managed to escape the radar of regulators.

When regrettable substitution occurs, different pillars on which the EU Regulatory scheme is based become distorted and altered. For example, if a hazardous chemical is replaced by an equally hazardous one, simply because the alternative has managed to make its way into the EU market without sufficient data being available, the 'No data, no market' paradigm is turned into 'No data, no problem' for the supplier of the alternative. In addition, when downstream users make the choice of sourcing an alternatively only on the basis that it is not classified by a supplier, the original scope of substitution, which is the replacement of a chemical by its 'Least Hazardous Alternative' is changed to replacement by the 'Least Regulated Alternative'.

This situation undermines the confidence of those companies that are committed to ensuring compliance with their obligations in terms of REACH and CLP, with the cost and efforts that this implies, who have to see how their competitors may get an advantage in the market while not having to incur in the burden that regulatory compliance requires. This creates a negative perception of the functioning of the EU regulatory scheme by responsible stakeholders, and it can also lead to unnecessary friction in the relevant supply chains between suppliers and customers.

EU Authorities should make all efforts to avoid these situations and to ensure a level playing field in the EU chemicals market, while providing regulatory certainty to the responsible suppliers, downstream users and workers handling potentially hazardous chemicals. Furthermore, situations in which companies have to act as 'the police' of competitors should also be avoided. 